\title{
Kidney Tumor Segmentation Based on U-Net and V-Net with Double Loss Function Training
}

\author{
Yi Lv ${ }^{1}$ and Junchen Wang ${ }^{1}$ \\ ${ }^{1}$ School of Mechanical Engineering and Automation, Beihang University, Beijing, 100191, \\ China. \\ dnkii@foxmail.com
}

\begin{abstract}
Medical image processing plays an increasingly important role in clinical diagnosis and treatment. Using the results of kidney CT image segmentation for three-dimensional reconstruction is an intuitive and accurate method for diagnosis. However, the traditional image segmentation algorithm has poor performance due to the large difference of noise between kidney and CT images, and the manual segmentation by doctors will take a very long time and is inefficient. In this paper, we propose an in-depth learning automatic segmentation method for kidney tumors, including preprocessing of training data, network model used in training process, loss function and post-processing, etc. The results show that the average dice of kidney with tumor was 0.97 in the test set.
\end{abstract}

Keywords: Medical Image Segmentation, Deep Learning, Neural Network.

\section{Introduction}

Segmentation and reconstruction of CT or MRI medical images is the main source of navigation data, i.e. anatomical structure of tissues and organs[1-4]. At present, the most commonly used method of medical image segmentation is still manual segmentation, which takes a long time and depends on the operator's experience and skills[5]. In recent years, breakthroughs have been made in the research of neural networks[69]. The deep learning technology based on neural networks can achieve fast segmentation, and effectively solve the problem of low accuracy and long time-consuming image segmentation[10]. In the field of medical image segmentation, the breakthrough of in-depth learning began with the introduction of Full Convolutional Neural Network (FCN), and another breakthrough of neural network architecture U-Net made it possible to achieve high-precision automatic segmentation of medical images. Long Jonathan et al.[11]

In this paper, we propose an in-depth learning automatic segmentation method for kidney tumors, including preprocessing of training data, network model used in training process, loss function and post-processing, etc. 


\section{Operational Process}

\subsection{Initial Segmentation Using U-NET}

Firstly, we divide the data set into two parts: 200 patients' CT sequences as training sets and 10 patients' CT sequences as test sets. Then we use the training set to train UNET directly for segmentation task, which is to roughly find the region of kidney. Figure 1 shows the results of segmentation, in which the results of segmentation have met our expectations.

As in (1), we use Binary Cross Entropy(BCE)[12] to measure the error between model output and label in medical image processing.

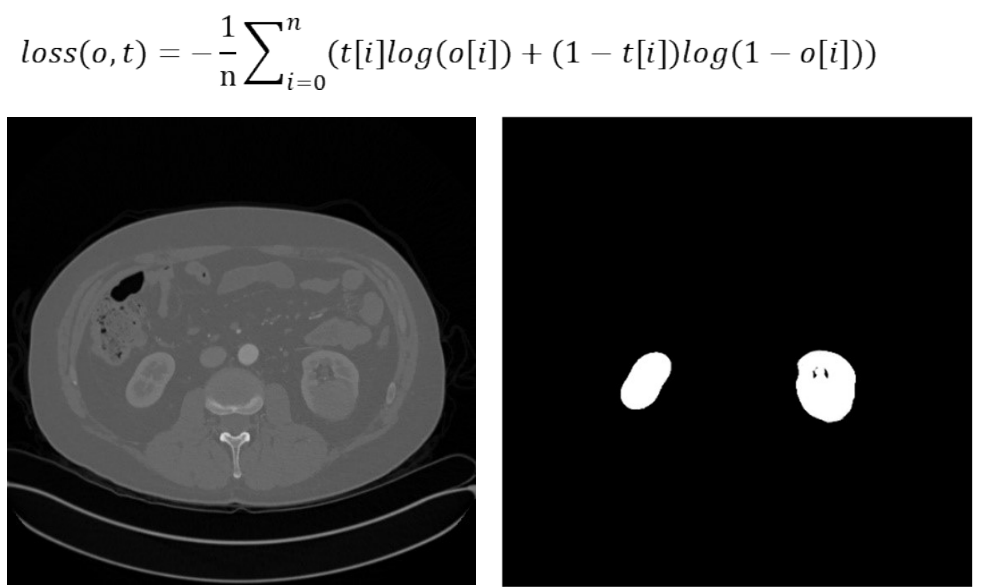

Fig. 1. The result of initial segmentation

\subsection{Fine Segmentation Using V-NET}

In this step, we will use the results of the previous step to calculate the area of the kidney and tailor it. As shown in Figure 2, we cut the mask part into a threedimensional sequence. In order to generate trainset for V-Net, we scale this threedimensional sequence to $128 * 128 * 64$ sequence. Then we use the segmentation result of training set to train V-Net to segment kidney and tumor. It is worth noting that during the training process, we tested several different loss functions and functions defined by ourselves to test the effect. Including weight cross-entropy, square deviation and Dice loss function. The results show that the square difference and Dice loss function are more suitable for the task of three-dimensional segmentation. At this stage, we still use the CT sequences of the first 200 patients as training set. After cutting, we obtained 390 sequences of $128 * 128 * 64$ containing kidneys. 

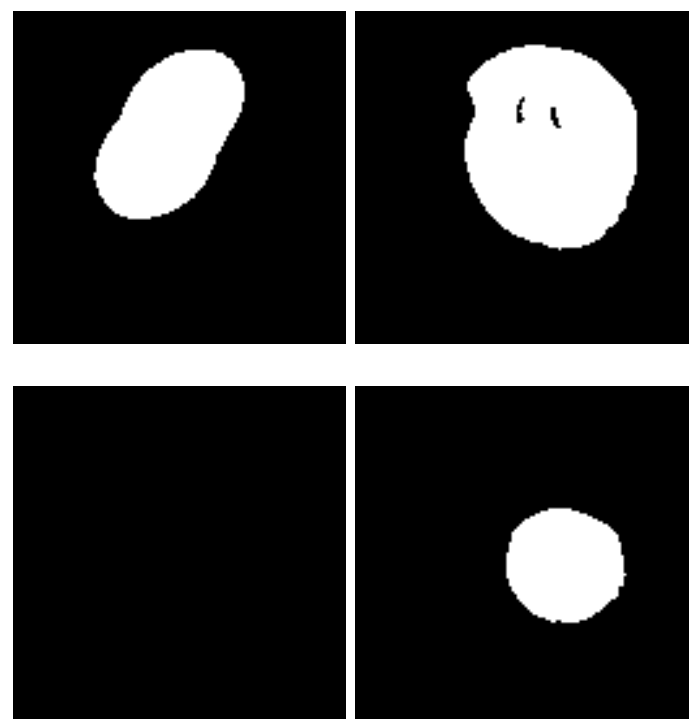

Fig. 2. The result of fine segmentation

\subsection{Restoring to Original CT Part}

After the first two steps, we can get a U-Net model which can segment the kidney from $512 * 512$ image, a $128 * 128 * 64$ three-dimensional sequence cut and scaled according to the U-Net result, a V-Net model which can segment the kidney from $128 * 128 * 64$ three-dimensional sequence, and a segmented and deformed kidney in $128 * 128 * 64$ sequence. In this step, we restore the segmentation result to the original CT image size. As shown in Figure 3, by comparison, it can be found that the segmentation results obtained by this method are better than direct segmentation in most cases.

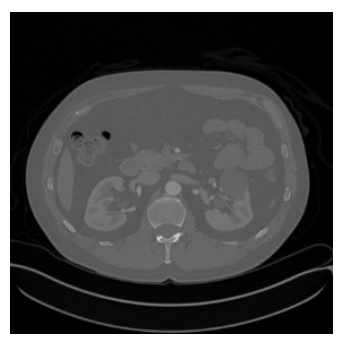

(a)

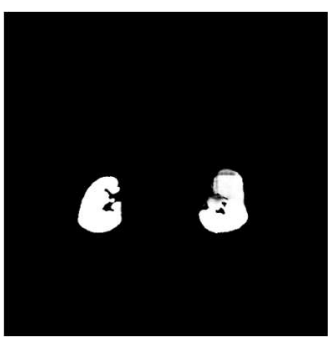

(b)

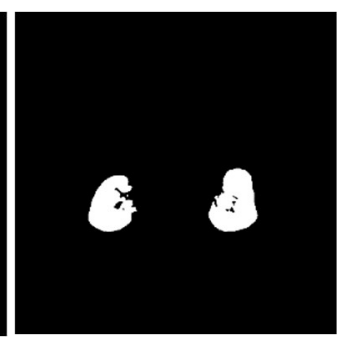

(c)

Fig. 3. Comparison of segmentation results. (a)CT image. (b)result of U-Net. (c)result of VNet. 


\subsection{Kidney Tumor Segmentation Model Training}

The V-Net model is also trained for the segmentation of kidney tumors. As in the second step, we regard the tumors as another label of $128 * 128 * 64$ sequence. The segmentation results of the model are restored to the original size by the same restoring method as in the third step.

\section{References}

[1] V. Couteaux, S. Si-Mohamed, R. Renard-Penna, O. Nempont, T. Lefevre, A. Popoff, G. Pizaine, N. Villain, I. Bloch, J. Behr, M.F. Bellin, C. Roy, O. Rouviere, S. Montagne, N. Lassau, L. Boussel, Kidney cortex segmentation in 2D CT with U-Nets ensemble aggregation, Diagn. Interv. Imaging 100(4) (2019) 211-217.

[2] Y. Yang, H. Jiang, Q. Sun, A Multiorgan Segmentation Model for CT Volumes via Full Convolution-Deconvolution Network, Biomed Research International (2017).

[3] Y. Fu, T.R. Mazur, X. Wu, S. Liu, X. Chang, Y. Lu, H.H. Li, H. Kim, M.C. Roach, L. Henke, D. Yang, A novel MRI segmentation method using CNN-based correction network for MRI-guided adaptive radiotherapy, Medical Physics 45(11) (2018) 5129-5137.

[4] C. Zhao, A. Carass, J. Lee, Y. He, J.L. Prince, Whole Brain Segmentation and Labeling from CT Using Synthetic MR Images, in: Q. Wang, Y. Shi, H.I. Suk, K. Suzuki (Eds.), Machine Learning in Medical Imaging2017, pp. 291-298.

[5] J. Li, S.a. Zhu, H. Bin, Medical image segmentation techniques, Sheng wu yi xue gong cheng xue za zhi $=$ Journal of biomedical engineering = Shengwu yixue gongchengxue zazhi 23(4) (2006) 891-4.

[6] E. Micheli-Tzanakou, Artificial Neural Networks: An Overview, Network-Computation in Neural Systems 22(1-4) (2011) 208-230.

[7] Y. LeCun, Y. Bengio, G. Hinton, Deep learning, Nature 521(7553) (2015) 436-444.

[8] J. Zhang, C. Zong, Deep Neural Networks in Machine Translation: An Overview, Ieee Intelligent Systems 30(5) (2015) 16-25.

[9] A. Zarandy, C. Rekeczky, P. Szolgay, L.O. Chua, Ieee, Overview of CNN Research: 25 Years History and the Current Trends, 2015 Ieee International Symposium on Circuits and Systems2015, pp. 401-404.

[10] D.Y. Kim, J.W. Park, Computer-aided detection of kidney tumor on abdominal computed tomography scans, Acta Radiologica 45(7) (2004) 791-795.

[11] E. Shelhamer, J. Long, T. Darrell, Fully Convolutional Networks for Semantic Segmentation, IEEE Trans. Pattern Anal. Mach. Intell. 39(4) (2017) 640-651.

[12] Y.D. Ma, Q. Liu, Z.B. Qian, Ieee, Automated image segmentation using improved PCNN model based on cross-entropy, 2004. 